SISSA/30/99/EP

\title{
On the relation between Green's functions of the SUSY theory with and without soft terms
}

\author{
Igor Kondrashuk円 \\ SISSA - ISAS and INFN, Sezione di Trieste, \\ Via Beirut 2-4, I-34013, Trieste, Italy
}

\begin{abstract}
We study possible relations between the full Green's functions of softly broken supersymmetric theories and the full Green's functions of rigid supersymmetric theories on the example of the supersymmetric quantum mechanics and find that algebraic relations can exist and can be written in a simple form. These algebraic relations between the Green's functions have been derived by transforming the path integral of the rigid theory. In this approach soft terms appear as the result of general changes of coordinates in the superspace.
\end{abstract}

\footnotetext{
${ }^{1}$ E-mail: ikond@sissa.it, on leave of absence from LNP, JINR, Dubna, Russia
} 
The renormalization of softly broken supersymmetric Yang-Mills theories in four space-time dimensions into the framework of the superfield technique and spurions method was studied from different points of view in Refs. ([1]-[6]). The soft terms were introduced as the finite series in terms of Grassmanian coordinates of the superspace. It results in dependence of renormalization constants of the theory on the Grassmanian coordinates. As it was understood, there are direct relations between the renormalization constants of the rigid theory - theory without soft terms - and the renormalization constants of its softly broken counterpart. The first investigation in

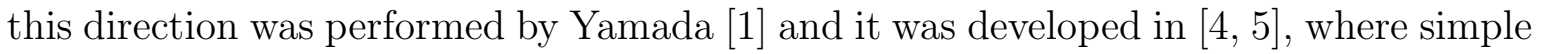
rules to calculate these constants starting from the well-known results for the rigid theory have been derived. These rules are realized in terms of differential operators acting in the coupling space of the rigid theory. In the Ref. [5] it was found that this approach is in good agreement with nonperturbative results [2]. The reason why these rules exist is that the soft breaking terms can be considered as background $x$-independent superfields [5, [0] in which our theory is embedded. These external superfields must be substituted into infinite parts of the Green's functions instead of the couplings of the rigid theory. The algebraic relations between the renormalizations constants are consequences of these substitutions [5].

However, it would be nice to have relations for the complete Green's functions of the theory that includes soft terms and for its counterpart without them. Here we find these relations for the case of the supersymmetric quantum mechanics as a simplest example where the method proposed below can work. The supersymmetric quantum mechanics was developed in ([7]-[9]) and its action is ?]

$$
S^{R}=\int d x d \theta d \bar{\theta}(D \Phi \bar{D} \Phi+V(\Phi))
$$

where $V$ is an arbitrary function of the real superfield $\Phi$,

$$
\Phi(x, \theta, \bar{\theta})=\phi(x)+i \theta \psi(x)+i \bar{\theta} \bar{\psi}(x)+\theta \bar{\theta} D(x), \quad \Phi^{\dagger}=\Phi .
$$

The Lagrangian (1) is the only thing of the supersymmetric quantum mechanics we need. In the rest of this letter we work with the path integral produced by this classical action. The Green's functions of it are referred in what follows as the rigid Green's functions:

$$
\begin{gathered}
G_{n}^{R}\left(x_{1}, x_{2}, \ldots, x_{n}, \theta_{1}, \ldots, \theta_{n}, \bar{\theta}_{1}, \ldots, \bar{\theta}_{n}\right)= \\
\left.\frac{\delta}{\delta J\left(x_{1}, \theta_{1}, \bar{\theta}_{n}\right)} \frac{\delta}{\delta J\left(x_{2}, \theta_{1}, \bar{\theta}_{1}\right)} \ldots \frac{\delta}{\delta J\left(x_{n}, \theta_{n}, \bar{\theta}_{n}\right)} Z^{R}[J]\right|_{J=0}, \\
Z^{R}[J]=\int \mathcal{D} \Phi \exp \left(S^{R}+\int d x d \theta d \bar{\theta} J \Phi\right) .
\end{gathered}
$$

Some comments about this expression are necessary. The Green's functions are local in the Grassmanian $\theta$-coordinates [10]. This result can be seen in the perturbation theory

\footnotetext{
${ }^{2}$ Here the superscript $R$ stands for "rigid"
} 
after performing the Grassmanian integration. However, we would like to keep all the Grassmanian coordinates in the above expression since all the following speculations are based on the path integral only. The external source $J$ is also real superfield, $J^{\dagger}=J$. The covariant derivatives in the action (11) are defined as

$$
D=\frac{\partial}{\partial \theta}-i \bar{\theta} \frac{\partial}{\partial x}, \quad \bar{D}=\frac{\overleftarrow{\partial}}{\partial \bar{\theta}}+i \overleftarrow{\theta} \frac{\partial}{\partial x}
$$

Polynomials in Grassmanian coordinates which are background $x$-independent superfields can stand in front of the terms of the action (11),

$$
\begin{gathered}
S^{S}=\int d x d \theta d \bar{\theta}\left(P_{1}(\theta, \bar{\theta}) D \Phi \bar{D} \Phi+P_{2}(\theta, \bar{\theta}) V(\Phi)\right), \\
P_{i}(\theta, \bar{\theta})=\alpha_{i}+i \beta_{i} \theta+i \bar{\beta}_{i} \bar{\theta}+\gamma_{i} \theta \bar{\theta}, \quad P_{i}^{\dagger}=P_{i} .
\end{gathered}
$$

Here $\alpha_{i}, \beta_{i}$, and $\gamma_{i}$ are arbitrary numbers, $\beta_{i}$ is Grassmanian. In this case we say that there is a soft supersymmetry breaking since if we do not transform charges in a proper way we have non-invariance of the component action under the supersymmetry transformations caused by the change of the coordinates in the superspace

$$
\begin{gathered}
x \rightarrow \exp (\varepsilon Q+\bar{Q} \bar{\varepsilon}) x, \quad \theta \rightarrow \theta+\varepsilon, \quad \bar{\theta} \rightarrow \bar{\theta}+\bar{\varepsilon} \\
Q=\frac{\partial}{\partial \theta}+i \bar{\theta} \frac{\partial}{\partial x}, \quad \bar{Q}=\frac{\overleftarrow{\partial}}{\partial \bar{\theta}}-i \overleftarrow{\theta} \frac{\partial}{\partial x} .
\end{gathered}
$$

Our purpose is to show a way in which the rigid Green's functions and the soft Green's functions,

$$
\begin{gathered}
G_{n}^{S}\left(x_{1}, x_{2}, \ldots, x_{n}, \theta_{1}, \ldots, \theta_{n}, \bar{\theta}_{1}, \ldots, \bar{\theta}_{n}\right)= \\
\left.\frac{\delta}{\delta J\left(x_{1}, \theta_{1}, \bar{\theta}_{1}\right)} \frac{\delta}{\delta J\left(x_{2}, \theta_{2}, \bar{\theta}_{2}\right)} \ldots \frac{\delta}{\delta J\left(x_{n}, \theta_{n}, \bar{\theta}_{n}\right)} Z^{S}[J]\right|_{J=0}, \\
Z^{S}[J]=\int \mathcal{D} \Phi \exp \left(S^{S}+\int d x d \theta d \bar{\theta} J \Phi\right)
\end{gathered}
$$

can be related. First of all, let us suppose that a general change of the superspace coordinates is made,

$$
x=x(\tilde{x}, \tilde{\theta}, \overline{\tilde{\theta}}), \quad \theta=\theta(\tilde{x}, \tilde{\theta}, \overline{\tilde{\theta}}), \quad \bar{\theta}=\bar{\theta}(\tilde{x}, \tilde{\theta}, \overline{\tilde{\theta}}),
$$

and we look for changes which satisfy the conditions

$$
\begin{gathered}
\bar{D}(\tilde{x}-i \tilde{\tilde{\theta}} \overline{\tilde{\theta}})=0, \quad D(\tilde{x}+i \tilde{\theta} \tilde{\tilde{\theta}})=0, \\
\bar{D} \tilde{\theta}=0, \quad D \overline{\tilde{\theta}}=0, \quad \tilde{x}^{\dagger}=\tilde{x}
\end{gathered}
$$

where we suppose that we know the reversed law of the transformation (四)

$$
\tilde{x}=\tilde{x}(x, \theta, \bar{\theta}), \quad \tilde{\theta}=\tilde{\theta}(x, \theta, \bar{\theta}), \quad \overline{\tilde{\theta}}=\overline{\tilde{\theta}}(x, \theta, \bar{\theta}) .
$$


The conditions (5) mean that the covariant derivative transforms as

$$
D=\frac{\partial}{\partial \theta}-i \bar{\theta} \frac{\partial}{\partial x} \rightarrow(D \tilde{\theta})\left(\frac{\partial}{\partial \tilde{\theta}}-i \overline{\tilde{\theta}} \frac{\partial}{\partial \tilde{x}}\right),
$$

and $\bar{D}$ transforms in the Hermitian conjugated way. The most general change of superspace coordinates that satisfy the condition (5) is

$$
\begin{gathered}
\tilde{x}=g(x)+i \bar{\mu} \theta f(x) \sqrt{\partial g(x)}+i \mu \bar{\theta} f(x) \sqrt{\partial g(x)}-\mu \bar{\mu} \partial f^{2}(x) \theta \bar{\theta} \\
\tilde{\theta}=\theta \sqrt{\partial g(x-i \theta \bar{\theta})}+\mu f(x-i \theta \bar{\theta}) \\
\overline{\tilde{\theta}}=\bar{\theta} \sqrt{\partial g(x+i \theta \bar{\theta})}+\bar{\mu} f(x+i \theta \bar{\theta}),
\end{gathered}
$$

where the functions $f$ and $g$ are arbitrary and $\mu$ is a Grassmanian dimensionful constant. The Berezinian of this change of coordinates is a long expression and we do not write it here for the brevity. Nevertheless, it is possible to see already in the law (7) that the $(\theta=\bar{\theta}=0)$-component of the Berezinian is equal to 1 for any functions $f$ and $g$. At the same time the factors $D \tilde{\theta}$ and $\bar{D} \overline{\tilde{\theta}}$ arising in (6) under the change of coordinates in front of the covariant derivatives in the kynetic term of the rigid action $S^{R}$ has the $\sqrt{\partial g}$ as its $(\theta=\bar{\theta}=0)$-component. Therefore, in order to have the independent on the space coordinate factor after the change is made we should take $g(x)=x$ and keep $f(x)$ arbitrary for a moment. Then the form of the above transformation is

$$
\begin{gathered}
\tilde{x}=x+i \bar{\mu} \theta f(x)+i \mu \bar{\theta} f(x)-\mu \bar{\mu} \partial f^{2}(x) \theta \bar{\theta} \\
\tilde{\theta}=\theta+\mu f(x-i \theta \bar{\theta}) \\
\overline{\tilde{\theta}}=\bar{\theta}+\bar{\mu} f(x+i \theta \bar{\theta}) .
\end{gathered}
$$

The change of coordinates reversed to $(8)$ is

$$
\begin{gathered}
x=\tilde{x}-i \bar{\mu} \tilde{\theta} f(\tilde{x})-i \mu \overline{\tilde{\theta}} f(\tilde{x})-\mu \bar{\mu} \partial f^{2}(\tilde{x}) \tilde{\theta} \tilde{\tilde{\theta}} \\
\theta=\tilde{\theta}\left(1+i \mu \bar{\mu} \partial f^{2}(\tilde{x})\right)-\mu f(\tilde{x}-i \tilde{\tilde{\theta}}) \\
\bar{\theta}=\overline{\tilde{\theta}}\left(1-i \mu \bar{\mu} \partial f^{2}(\tilde{x})\right)-\bar{\mu} f(\tilde{x}+i \tilde{\theta} \overline{\tilde{\theta}}) .
\end{gathered}
$$

By the explicit calculation we see that the Berezinian of this change is equal to 1 for any function $f$. To have a common factor arising in (6) as $x$-independent background superfield, we should choose $f(x)=x$. In this case, the transformation (8) is

$$
\begin{gathered}
\tilde{x}=x(1+i \bar{\mu} \theta+i \mu \bar{\theta}-2 \mu \bar{\mu} \theta \bar{\theta})=x(1-i \bar{\mu} \theta-i \mu \bar{\theta})^{-1} \\
\tilde{\theta}=\theta+\mu(x-i \theta \bar{\theta}) \\
\overline{\tilde{\theta}}=\bar{\theta}+\bar{\mu}(x+i \theta \bar{\theta}),
\end{gathered}
$$

and the reversed transformation is

$$
\begin{gathered}
x=\tilde{x}(1+i \bar{\mu} \tilde{\theta}+i \mu \overline{\tilde{\theta}})^{-1} \\
\theta=(\tilde{\theta}-\mu(\tilde{x}-i \tilde{\theta} \tilde{\tilde{\theta}})) \exp (-2 i \bar{\mu} \tilde{\theta}) \\
\bar{\theta}=(\overline{\tilde{\theta}}-\bar{\mu}(\tilde{x}+i \tilde{\tilde{\theta}})) \exp (-2 i \mu \overline{\tilde{\theta}}) .
\end{gathered}
$$


The factors $D \tilde{\theta}$ and $\mathcal{D} \overline{\tilde{\theta}}$ that come from (6) are

$$
\begin{aligned}
D \tilde{\theta}=\exp (2 i \mu \bar{\theta}) & =\exp (2 i \mu[\overline{\tilde{\theta}}-\bar{\mu}(\tilde{x}+i \tilde{\theta} \overline{\tilde{\theta}})]) \\
\bar{D} \overline{\tilde{\theta}}=\exp (2 i \bar{\mu} \theta) & =\exp (2 i \bar{\mu}[\tilde{\theta}-\mu(\tilde{x}-i \tilde{\tilde{\theta}})]) .
\end{aligned}
$$

Therefore, the common factor that is due to the transformation of the derivatives (6) is

$$
D \tilde{\theta} \bar{D} \overline{\tilde{\theta}}=\exp (2 i \mu \overline{\tilde{\theta}}+2 i \bar{\mu} \tilde{\theta}+4 \mu \bar{\mu} \tilde{\tilde{\theta}} \overline{\tilde{\theta}})=1+2 i \mu \overline{\tilde{\theta}}+2 i \bar{\mu} \tilde{\theta}
$$

We can make the change of coordinates (11) in the rigid action $S^{R}$ of the path integral $Z^{R}$ :

$$
\begin{gathered}
Z^{R}[J]=\int \mathcal{D} \Phi \exp \left(\int d x d \theta d \bar{\theta}[D \Phi \bar{D} \Phi+V(\Phi)+J \Phi]\right)= \\
=\int \mathcal{D} \tilde{\Phi} \exp \left(\int d x d \theta d \bar{\theta}[(1+2 i \mu \bar{\theta}+2 i \bar{\mu} \theta) D \tilde{\Phi} \bar{D} \tilde{\Phi}+V(\tilde{\Phi})+\tilde{J} \tilde{\Phi}]\right)=Z^{S}[\tilde{J}]
\end{gathered}
$$

where the definitions are used:

$$
\begin{aligned}
& \tilde{\Phi}(x, \theta, \bar{\theta})=\Phi\left(\frac{x}{1+i \bar{\mu} \theta+i \mu \bar{\theta}}, \quad(\theta-\mu(x-i \theta \bar{\theta})) e^{-2 i \bar{\mu} \theta}, \quad(\bar{\theta}-\bar{\mu}(x+i \theta \bar{\theta})) e^{-2 i \mu \bar{\theta}}\right) \\
& \tilde{J}(x, \theta, \bar{\theta}) \equiv J\left(\frac{x}{1+i \bar{\mu} \theta+i \mu \bar{\theta}}, \quad(\theta-\mu(x-i \theta \bar{\theta})) e^{-2 i \bar{\mu} \theta}, \quad(\bar{\theta}-\bar{\mu}(x+i \theta \bar{\theta})) e^{-2 i \mu \bar{\theta}}\right)
\end{aligned}
$$

As one can see the second part of (14) is the path integral of the theory with soft supersymmetry breaking terms that correspond according to (2) to

$$
P_{1}(\theta, \bar{\theta})=1+2 i \mu \bar{\theta}+2 i \bar{\mu} \theta, \quad P_{2}(\theta, \bar{\theta})=1
$$

We can expand this soft path integral $Z^{S}$ in terms of the external source $\tilde{J}$ and the coefficient functions of this expansion are in fact the Green's functions of the soft theory

$$
\begin{gathered}
Z^{S}[\tilde{J}]=\sum_{n} \int d x_{1} d x_{2} \ldots d x_{n} d \theta_{1} \ldots d \theta_{n} d \bar{\theta}_{1} \ldots d \bar{\theta}_{n} \frac{1}{n !} G_{n}^{S}\left(x_{1}, x_{2}, \ldots, x_{n}, \theta_{1}, \theta_{2}, \ldots\right. \\
\left.\ldots, \theta_{n}, \bar{\theta}_{1}, \bar{\theta}_{2}, \ldots, \bar{\theta}_{n}\right) \tilde{J}\left(x_{1}, \theta_{1}, \bar{\theta}_{1}\right) \tilde{J}\left(x_{2}, \theta_{2}, \bar{\theta}_{2}\right) \ldots \tilde{J}\left(x_{n}, \theta_{n}, \bar{\theta}_{n}\right)
\end{gathered}
$$

Now we can make the change of coordinates back and restore the original sources $J$. It means that by changing the coordinates in the superspace as in (10) for each of $x_{i}$ we have instead of (16) the expression

$$
\begin{gathered}
Z^{S}[\tilde{J}[J]]=\sum_{n} \int d x_{1} d x_{2} \ldots d x_{n} d \theta_{1} \ldots d \theta_{n} d \bar{\theta}_{1} \ldots d \bar{\theta}_{n} \frac{1}{n !} * \\
G_{n}^{S}\left(\frac{x_{1}}{1-i \bar{\mu} \theta_{1}-i \mu \bar{\theta}_{1}}, \ldots, \frac{x_{n}}{1-i \bar{\mu} \theta_{n}-i \mu \bar{\theta}_{n}}, \theta_{1}+\mu\left(x_{1}-i \theta_{1} \bar{\theta}_{1}\right), \ldots,\right. \\
\left.\ldots, \theta_{n}+\mu\left(x_{n}-i \theta_{n} \bar{\theta}_{n}\right), \bar{\theta}_{1}+\bar{\mu}\left(x_{1}+i \theta_{1} \bar{\theta}_{1}\right), \ldots, \bar{\theta}_{n}+\bar{\mu}\left(x_{n}+i \theta_{n} \bar{\theta}_{n}\right)\right) * \\
* J\left(x_{1}, \theta_{1}, \bar{\theta}_{1}\right) J\left(x_{2}, \theta_{2}, \bar{\theta}_{2}\right) \ldots J\left(x_{n}, \theta_{n}, \bar{\theta}_{n}\right) .
\end{gathered}
$$


As one can see from (14), the expansion (17) is at the same time the expansion of the $Z^{R}[J]$ in terms of the external source of the rigid theory $J$. Therefore, the following equality takes place

$$
\begin{gathered}
G_{n}^{R}\left(x_{1}, x_{2}, \ldots, x_{n}, \theta_{1}, \theta_{2}, \ldots, \theta_{n}, \bar{\theta}_{1}, \bar{\theta}_{2}, \ldots, \bar{\theta}_{n}\right)= \\
G_{n}^{S}\left(\frac{x_{1}}{1-i \bar{\mu} \theta_{1}-i \mu \bar{\theta}_{1}}, \ldots, \frac{x_{n}}{1-i \bar{\mu} \theta_{n}-i \mu \bar{\theta}_{n}}, \theta_{1}+\mu\left(x_{1}-i \theta_{1} \bar{\theta}_{1}\right), \ldots,\right. \\
\left.\ldots, \theta_{n}+\mu\left(x_{n}-i \theta_{n} \bar{\theta}_{n}\right), \bar{\theta}_{1}+\bar{\mu}\left(x_{1}+i \theta_{1} \bar{\theta}_{1}\right), \ldots, \bar{\theta}_{n}+\bar{\mu}\left(x_{n}+i \theta_{n} \bar{\theta}_{n}\right)\right) .
\end{gathered}
$$

Having used the substitutions (11) we transform the equation (18) to the form

$$
\begin{gathered}
G_{n}^{S}\left(x_{1}, x_{2}, \ldots, x_{n}, \theta_{1}, \theta_{2}, \ldots, \theta_{n}, \bar{\theta}_{1}, \bar{\theta}_{2}, \ldots, \bar{\theta}_{n}\right)= \\
G_{n}^{R}\left(\frac{x_{1}}{1+i \bar{\mu} \theta_{1}+i \mu \bar{\theta}_{1}}, \ldots, \frac{x_{n}}{1+i \bar{\mu} \theta_{n}+i \mu \bar{\theta}_{n}},\left(\theta_{1}-\mu\left(x_{1}-i \theta_{1} \bar{\theta}_{1}\right)\right) e^{-2 i \bar{\mu} \theta_{1}}, \ldots,\right. \\
\ldots,\left(\theta_{n}-\mu\left(x_{n}-i \theta_{n} \bar{\theta}_{n}\right)\right) e^{-2 i \bar{\mu} \theta_{n}},\left(\bar{\theta}_{1}-\bar{\mu}\left(x_{1}+i \theta_{1} \bar{\theta}_{1}\right)\right) e^{-2 i \mu \bar{\theta}_{1}}, \ldots, \\
\left.\ldots,\left(\bar{\theta}_{n}-\bar{\mu}\left(x_{n}+i \theta_{n} \bar{\theta}_{n}\right)\right) e^{-2 i \mu \bar{\theta}_{n}}\right) .
\end{gathered}
$$

Thus, the final result is that the theory with soft supersymmetry breaking terms (2) in the form (15) is equivalent to the rigid theory (11) in the sense of the relation (19) between their Green's functions. It looks like something surprising that we could relate soft and rigid theories, but the explanation is if we amount couplings to background $x$-independent superfields we have their transformations at the level of the component action which are the reflection of the change of coordinates in the superspace (3). Hence, if we treat couplings of theory at the component level as components of an external background multiplet, we have no supersymmetry breaking since we include rigid couplings and soft couplings in the supersymmetry transformation (3). Nevertheless, we can create soft terms under general changes of the coordinates in the superspace.

I thank A. Masiero for stimulating discussions. The investigation is supported by INFN.

\section{References}

[1] Y. Yamada, Phys.Rev. D50(1994)3537.

[2] J. Hisano, M. Shifman, hep-th/9705417, Phys.Rev. D56(1997)5475

[3] G.F. Giudice and R. Rattazzi, hep-ph/9706540, Nucl.Phys. B511(1998)25

[4] I. Jack and D.R.T. Jones, hep-ph/9709364, Phys.Lett. B415(1997)383

[5] L.V. Avdeev, D.I. Kazakov, and I.N. Kondrashuk, hep-ph/9709397, Nucl.Phys. B510(1998)289-312 
[6] N. Arkani-Hamed, G.F. Giudice, M.A. Luty and R. Rattazzi, hep-ph/9803290, Phys.Rev. D58(1998) 115005

[7] E. Witten, Nucl.Phys. B188(1981)513

[8] E. Witten, Nucl.Phys. B202(1982)253

[9] F. Cooper and B. Freedman, Ann.Phys. 146(1983)262

[10] P. West, "Introduction to Supersymmetry and Supergravity", World Scientific, 1986. 